\title{
Confirmation of the role of pathogenic SMAD6 variants in bicuspid aortic valve-related aortopathy
}

\author{
Ilse Luyckx ${ }^{1}$ - Gretchen MacCarrick ${ }^{2}$ - Marlies Kempers ${ }^{3}$ - Josephina Meester ${ }^{1}$ - Céline Geryl ${ }^{1}$ - Olivier Rombouts ${ }^{1}$. \\ Nils Peeters ${ }^{1}$ - Charlotte Claes $^{1} \cdot$ Nele Boeckx ${ }^{1}$ - Natzi Sakalihasan ${ }^{4}$ - Adeline Jacquinet $^{5}$ - Alexander Hoischen ${ }^{3,6,7}$. \\ Geert Vandeweyer ${ }^{1} \cdot$ Sarah Van Lent $^{1} \cdot$ Johan Saenen $^{8} \cdot$ Emeline Van Craenenbroeck $^{8} \cdot$ Janneke Timmermans $^{9}$.

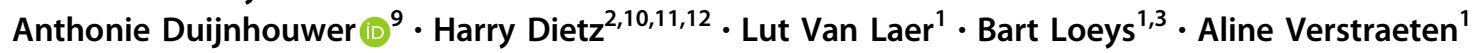

Received: 31 October 2018 / Revised: 22 January 2019 / Accepted: 24 January 2019 / Published online: 22 February 2019

(c) European Society of Human Genetics 2019

\begin{abstract}
Progressive dilatation of the thoracic aorta leads to thoracic aortic aneurysm (TAA), which is often asymptomatic but predisposes to lethal aortic dissections and ruptures. TAA is a common complication in patients with bicuspid aortic valve (BAV). Recently, rare loss-of-function SMAD6 variants were shown to contribute significantly to the genetic aetiology of BAV/TAA. Intriguingly, patients with craniosynostosis have also been reported to be explained molecularly by similar lossof-function SMAD6 variants. While significantly reduced penetrance of craniosynostosis has been reported for the SMAD6 variants as such, near-complete penetrance is reached upon co-occurrence with a common BMP2 SNP risk allele. Here, we report on the results of a SMAD6-variant analysis in 473 unrelated non-syndromic TAA patients, of which the SMAD6positive individuals were also studied for the presence of the $B M P 2$ risk allele. Although only $14 \%$ of the TAA patients also presented BAV, all novel likely pathogenic SMAD6 variants $(N=7)$ were identified in BAV/TAA individuals, further establishing the role of SMAD6 variants to the aetiology of BAV/TAA and revealing limited contribution to TAA development in patients with a tricuspid aortic valve. Familial segregation studies confirmed reduced penetrance (82\%) and variable clinical expressivity, with coarctation of the aorta being a common comorbidity. None of our six $B M P 2+/ S M A D 6+$ patients presented with craniosynostosis. Hence, the proposed digenic model for craniosynostosis was not supported in the presented BAV/TAA cohort, suggesting that additional factors are at play. Finally, our data provide improved insights into the clinical spectrum of SMAD6-related BAV/TAA and has important implications for molecular diagnostics.
\end{abstract}

\section{Introduction}

Thoracic aortic aneurysm (TAA) is characterized by progressive dilatation of the thoracic aorta. Untreated TAA can lead to life-threatening aortic dissection and/or rupture [1]. TAA can present as an isolated condition or as part of connective tissue disorders such as Marfan syndrome and

These authors contributed equally: Bart Loeys, Aline Verstraeten

Supplementary information The online version of this article (https:// doi.org/10.1038/s41431-019-0363-z) contains supplementary material, which is available to authorized users.

Aline Verstraeten

aline.verstraeten@uantwerpen.be

Extended author information available on the last page of the article
Loeys-Dietz syndrome [2]. It is also prevalent in individuals with bicuspid aortic valve (BAV), i.e. the most common congenital heart disorder characterized by two aortic valve leaflets instead of the normal three (tricuspid aortic valve, TAV) [3]. At least $10-20 \%$ of BAV patients develop TAA at some stage in life and BAV individuals have an eightfold increased risk of aortic dissection, typically occurring at young age $[4,5]$. Besides TAA, patients with BAV are at increased risk for aortic valve stenosis and coarctation (CoA) [5].

Therapies capable of preventing, stopping, or reversing TAA formation are not available yet. Early diagnosis and continuous surveillance of at-risk individuals thus serve as life-saving procedures [3]. The availability of genetic testing would be extremely useful to identify pre-symptomatic individuals [6], but causal variants in the currently known TAA genes explain only about one-quarter of TAA patients 
[7]. A molecular explanation is identified in far fewer patients with BAV/TAA [8]. Hence, acquisition of improved insights into the genetic basis of TAA is indispensable for improved risk stratification.

Over the past couple of years, genetic variability in SMAD6 has been reported to impinge on the risk of congenital heart disease (CHD) [9-11]. SMAD6 encodes a negative regulator of the bone morphogenetic protein (BMP) pathway [12]. In 2012, Tan et al. [11] reported heterozygous SMAD6 variants in two sporadic BAV patients either combined with mild-to-moderate aortic valve stenosis or with CoA. In a study of 2871 paediatric patients with CHD, nine SMAD6 (likely) pathogenic variants were identified in patients presenting with tetralogy of Fallot, hypoplastic left heart syndrome, dextro-transposition of the great arteries, CoA, or BAV [10]. In 2017, rare SMAD6 variants were also shown to be significantly enriched in BAV/TAA cases, explaining $2.5 \%$ of patients [9]. The variant spectrum of SMAD6-related cardiovascular disease encompasses frameshift, nonsense and MH1/MH2-domain locating missense variants as well as an in-frame deletion (Suppl. Table 1). Prior segregation analysis, performed in two BAV/TAA families, revealed variable expressivity (BAV/TAA, TAV/TAA and CoA) and incomplete penetrance of TAA (at ages 28 and 39) [9].

Intriguingly, rare SMAD6 variants (i.e. truncating and MH1/MH2-located missense variants) have also been reported to cause non-syndromic midline craniosynostosis (Suppl. Table 1) [13, 14]. Craniosynostosis is the most common congenital craniofacial birth defect and results from the premature fusion of the cranial sutures. Whereas incomplete penetrance of craniosynostosis was observed for the SMAD6 variants as such, near-complete penetrance was reached upon co-occurrence with a common variant near $B M P 2$ (rs1884302) [13, 14]. Cardiovascular abnormalities were not reported in the SMAD6-related craniosynostosis patients, even not in the patient who carried the identical SMAD6 variant (p.(Pro152Profs*27), c.455_461del) found in a BAV/TAA patient $[9,14]$.

Because prior segregation analysis confirmed the presence of a SMAD6 variant in a TAA patient with a normal aortic valve, we interrogated the spectrum of phenotypical variability of SMAD6-positive patients, irrespective of the co-occurrence with BAV, in a cohort of 473 TAA patients. Secondly, since near-complete penetrance for craniosynostosis has been reported to be reached by the cooccurrence of a $B M P 2$ risk allele (rs1884302, minor allele frequency $(\mathrm{MAF}) \mathrm{C}$-allele $=34 \%)$ and a SMAD6 variant, we also genotyped this specific $B M P 2$ variant in the SMAD6-variant carriers presenting with cardiovascular disease. We anticipated the near absence of the $B M P 2$ single nucleotide polymorphism (SNP) risk allele in our SMAD6-positive patients.

\section{Material and methods}

\section{Study cohort}

The patient population consisted of 473 non-syndromic TAA patients ascertained from three different research centres: Radboud University Medical Center, Nijmegen, the Netherlands; Center of Medical Genetics, University of Antwerp and Antwerp University Hospital, Antwerp, Belgium; and Institute of Genetic Medicine, Johns Hopkins University, Baltimore, MD, USA. All TAA patients were negative for mutations in known TAA genes. Of these, 65 had BAV. All patients had a $Z$-score exceeding 3 $[15,16]$ when height and weight were available, or an aortic diameter of at least $40 \mathrm{~mm}$ at the level of the sinus of Valsalva or ascending aorta. The number of aortic valve leaflets and the aortic dimensions were determined using echocardiography, computed tomography, or magnetic resonance imaging. A positive family history was defined as having at least one first- or second-degree relative with BAV and/or TAA. The study was approved by the local ethics committees and all study participants gave informed consent at the respective samplecontributing centres.

\section{SMAD6 targeted resequencing and variant validation}

Whole blood- or saliva-derived genomic DNA (gDNA) was enriched for all coding SMAD6 exons (RefSeq transcript NM_005585.4) using centre-dependent TAA and/or CHD gene panels (HaloPlex Target Enrichment System [17]; single molecule Molecular Inversion Probes (smMIP) [18]; Agilent SureSelectXT Human All Exon $50 \mathrm{Mb}$; Invitae Aortopathy Comprehensive Panel). Subsequent sequencing was performed on an Illumina NextSeq500 or HiSeq1500 instrument $(2 \times 150$ bp or $2 \times 100$ bp paired-end). Regions of insufficient coverage were complemented with Sanger sequencing as previously described [9]. The sequences of the primers are listed in Suppl. Table 2.

We selected for rare $(\mathrm{MAF} \leq 0.1 \%$ in gnomAD [19] r2.0.2) heterozygous non-synonymous coding and splicesite ( $\pm 10 \mathrm{bp}$ from exon-intron boundaries) SMAD6 variants after applying quality parameters (coverage: $\geq 10 \times$, allelic balance: $0.25-0.85$ ). Pathogenicity programmes such as Combined Annotation Dependent Depletion (CADD, v1.4) [20], MutationTaster [21], SIFT [22] and PolyPhen-2 [23] were used to evaluate variant deleteriousness, and Alamut Visual 2.8.0 software (Rouen, France) was used to predict altered splicing. In addition, we selected high-confidence copy number variations with a low frequency in healthy individuals $(\mathrm{MAF} \leq 0.1 \%$ in the Database of Genomic Variants (DGV)) from the available gene panel sequencing 
a

Family 1

(chr15:g.(66825594_67400490)

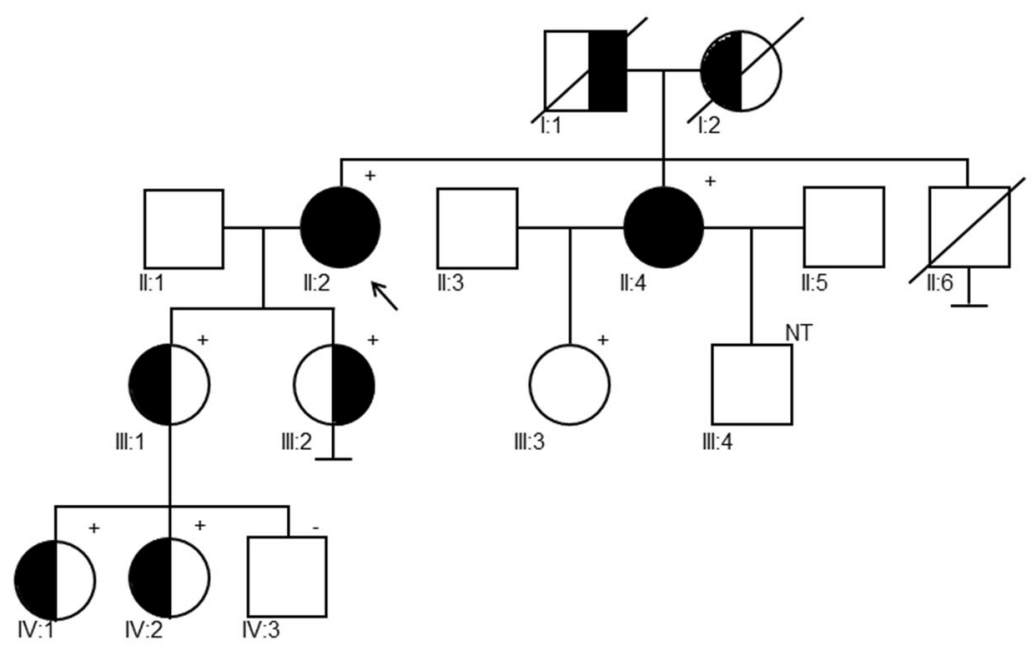

Family 4

p. $\left(\operatorname{Trp} 14^{\star}\right)$

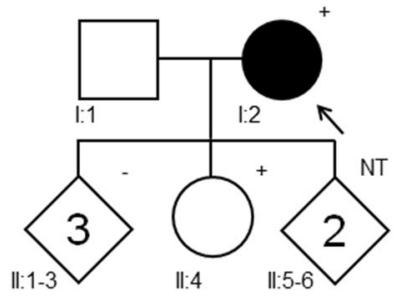

Family 5

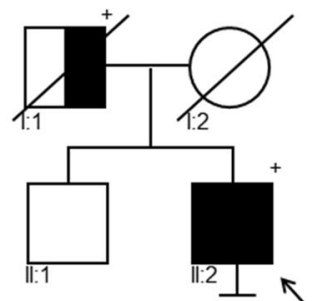

p.(His265Profs ${ }^{274}$ )
Family 2

p. (Pro257Leu) $)^{1} \&$ p.(Arg231Gly $)^{2}$

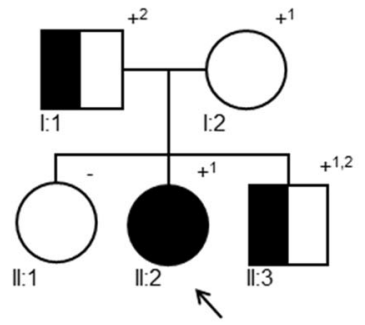

Family 3

p. $(\text { Arg231Pro })^{1} \&$ p. $(\text { Ala335Glu })^{2}$

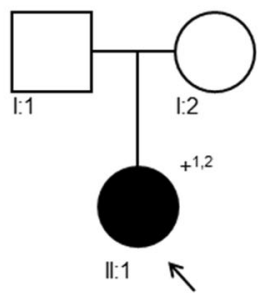

p.(Gly204Ala)

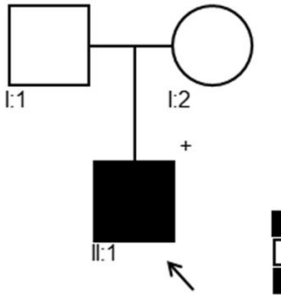

Heart defects

Vascular defects

Cardiovascular defects

b

\begin{tabular}{|c|c|c|c|c|c|c|c|c|}
\hline $\begin{array}{l}\text { Chromosomal } \\
\text { location (hg19) }\end{array}$ & $\begin{array}{l}\text { Nucleotide } \\
\text { change }\end{array}$ & $\begin{array}{l}\text { Protein } \\
\text { change }\end{array}$ & $\begin{array}{l}\text { Protein } \\
\text { domain }\end{array}$ & PolyPhen-2 & $\begin{array}{l}\text { Mutation } \\
\text { Taster2 }\end{array}$ & SIFT & CADD & gnomAD \\
\hline chr15:66995638 & c. $42 \mathrm{G}>\mathrm{A}$ & p. $\left(\operatorname{Trp} 14^{*}\right)$ & - & NA & $\begin{array}{l}\text { Disease } \\
\text { causing }\end{array}$ & NA & 46 & Absent \\
\hline chr15:66996207 & c. $611 \mathrm{G}>\mathrm{C}$ & p.(Gly204Ala) & MH1 & Benign & $\begin{array}{l}\text { Disease } \\
\text { causing }\end{array}$ & Tolerated & 21.3 & Absent \\
\hline chr15:66996287 & c. $691 \mathrm{C}>\mathrm{G}$ & p.(Arg231Gly) & MH1 & $\begin{array}{l}\text { Probably } \\
\text { Damaging }\end{array}$ & $\begin{array}{l}\text { Disease } \\
\text { causing }\end{array}$ & Damaging & 33 & Absent \\
\hline chr15:66996288 & c. $692 \mathrm{G}>\mathrm{C}$ & p.(Arg231Pro) & MH1 & $\begin{array}{l}\text { Probably } \\
\text { Damaging }\end{array}$ & $\begin{array}{l}\text { Disease } \\
\text { causing }\end{array}$ & Damaging & 29.9 & Absent \\
\hline chr15:66996390 & c.794del & $\begin{array}{l}\text { p.(His265Pro*fs } \\
\text { 274) }\end{array}$ & MH1 & NA & $\begin{array}{l}\text { Disease } \\
\text { causing }\end{array}$ & NA & NA & Absent \\
\hline chr15:67073386 & c. $1004 C>A$ & p.(Ala335Glu) & $\mathrm{MH} 2$ & $\begin{array}{l}\text { Probably } \\
\text { Damaging }\end{array}$ & $\begin{array}{l}\text { Disease } \\
\text { causing }\end{array}$ & Damaging & 30 & Absent \\
\hline $\begin{array}{l}\text { Chromosomal } \\
\text { location (hg19) }\end{array}$ & $\begin{array}{l}\text { Copy } \\
\text { Number }\end{array}$ & \multicolumn{2}{|c|}{ Min.-Max. size (kb) } & DGV & \multicolumn{4}{|l|}{ Involved genes } \\
\hline $\begin{array}{l}\text { chr15:g.(6682559 } \\
\text { 4_67400490) }\end{array}$ & 1 & $575-60$ & & Absent & \multicolumn{4}{|c|}{$\begin{array}{l}\text { LCTL, LINC01169, LINC02206, LOC102723493, SMAD6, } \\
\text { SMAD3, ZWILCH }\end{array}$} \\
\hline
\end{tabular}

c

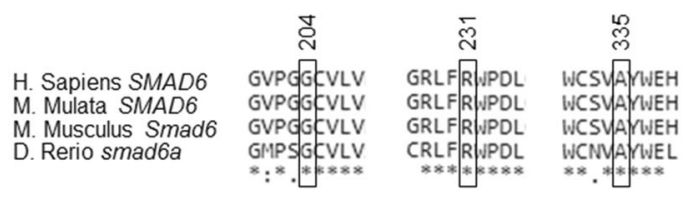

data. All variants of interest were validated with an independent technique (i.e. Sanger sequencing or SNPbased microarray). Segregation analysis was performed upon availability of gDNA of relatives. Variants listed in Fig. 1b are submitted to ClinVar (SCV000854413SCV000854420). And these variants were classified using 
Fig. 1 Variant analysis. a Pedigrees of the families with their respective SMAD6 variant. b Overview of the novel SMAD6 deletion and next-generation sequencing variants in our non-syndromic TAA cohort, and their respective annotations. Prediction programmes were used to evaluate their pathogenic effect (PolyPhen-2, MutationTaster2, SIFT (Sorting Intolerant From Tolerant) and CADD (Combined Annotation Dependent Depletion)). All variants are absent in gnomAD database, and the deletion was not reported in the Database of Genomic Variants (DGV). c Conservation of specific residues among species. (Reference build: GRCh37; RefSeq: NM_005585.4)

guidelines of the American College of Medical Genetics [24] (Suppl. Table 3).

\section{BMP2 rs1884302 genotyping}

All SMAD6-positive patients identified in this cohort and previously published by Gillis et al. [9] were genotyped for the $B M P 2$ rs 1884302 risk allele 'C' using Sanger sequencing as previously described [9].

\section{Results}

\section{Phenotypical characterization of patient cohort}

In total, 473 unrelated non-syndromic TAA patients were screened for genetic variability in SMAD6. The clinical and molecular characteristics of the study participants are summarized in Table 1 . The average age at inclusion was $53 \pm 17$ years and $70 \%$ were male, complying with the known 2:1 male preponderance of TAA [25]. In 14\% of the TAA patients $(N=65)$ the presence of BAV was noted. A positive family history was known for $30 \%$ of patients, whereas the family history was negative or unknown for $70 \%$ of the individuals.

\section{Contribution of likely pathogenic SMAD6 variants to the genetic aetiology of BAV/TAA and TAV/TAA}

We identified seven novel likely pathogenic SMAD6 variants in 473 non-syndromic TAA patients, i.e. $1.5 \%$ of the patient population (Fig. 1, Table 1). All SMAD6 variants were identified in BAV/TAA probands $(N=7 / 65,10.8 \%)$ (Table 2).

The first variant was a heterozygous deletion (hg19 chr15:g.(66,817,545_66,825,594)_

$\left(67,400,490 \_67,418,205\right)$ del) spanning the complete coding sequence of SMAD6 and exon 1 of SMAD3 (Fig. 1, Table 2). The cardiovascular features of the 67-year-old female proband (1-II:2) included BAV with severe valve stenosis and aortic regurgitation, an aortic root dilatation of $47 \mathrm{~mm}$, and a history of type A dissection extending to the region between the superior mesenteric artery and renal
Table 1 Characteristics of the non-syndromic TAA patient cohort

\begin{tabular}{ll}
\hline & Cohort $(N=473)(\%)$ \\
\hline $\begin{array}{l}\text { Average age (years) } \\
\text { Gender }\end{array}$ & $52.5 \pm 16.5$ \\
$\quad$ Male & $332(70.2)$ \\
$\quad$ Female & $141(29.8)$ \\
BAV & $65(13.7)$ \\
Family history & \\
$\quad$ Familial & $141(29.8)$ \\
$\quad$ Unknown & $332(70.2)$ \\
\hline
\end{tabular}

$C H D$ congenital heart disease, $B A V$ bicuspid aortic valve. $T A A$ thoracic aortic aneurysm

${ }^{\text {a}}$ Excluding four patients with an unspecified valve replacement (possibly: $14.6 \%$ of the cohort with BAV)

arteries. In addition, she presented with an aneurysm of the arteria basilaris, subconjunctival haemorrhages and mitral valve prolapse (MVP). Emergent repair for the dissection was performed at age 64. Six months later, the proband received Bentall aortic root repair with prosthetic aortic valve. Non-cardiovascular manifestations comprised diverticulitis, joint arthralgia, ventral hernia, anaemia, fatty liver, splenomegaly (due to a post-dissection splenic infarct) as well as Crohn's disease and auto-immune hepatitis. She also tested positive for the antinuclear antibodies. The father of the proband (1-I:1) died of stroke at age 84 and the mother (1-I:2) died of heart failure at age 86. None of the parents were known to have aneurysmal disease but gDNA is not available for genetic testing. The proband's two variantcarrying children presented with either TAV, mild-tomoderate aortic regurgitation and normal aortic diameters (1-III:1, 40-year-old) or a borderline ascending aortic dilatation (37 mm) (1-III:2, 38-year-old), respectively. Two (1IV:1, age 9; 1-IV:2, age 7) of the three grandchildren tested positive for the familial SMAD6 variant and presented with cardiac features such as pulmonary valve stenosis (1-IV:1) and patent foramen ovale (1-IV:2). Both individuals had TAV and normal aortic diameters. The variant-positive sister of the proband (1-II:4, age 65) had a type A aortic dissection extending to the abdominal part of the aorta at age 64. The thoracic aorta showed mild-to-moderate atherosclerosis. She also presented with patent foramen ovale and moderate mitral regurgitation with MVP, but a normal aortic valve. Non-cardiovascular features included umbilical hernia, scoliosis, cervical spine fusion (compressed vertebrae), arthritis (with surgery for feet and thumbs). Echocardiography in the variant-carrying niece of the proband (1-III:3, age 45) was normal. She has a history of arthritis, migraines and melanoma. The cardiovascular status of the 41-year-old nephew of the proband (1-III:4) is unknown, but he is also known with arthrosis. 


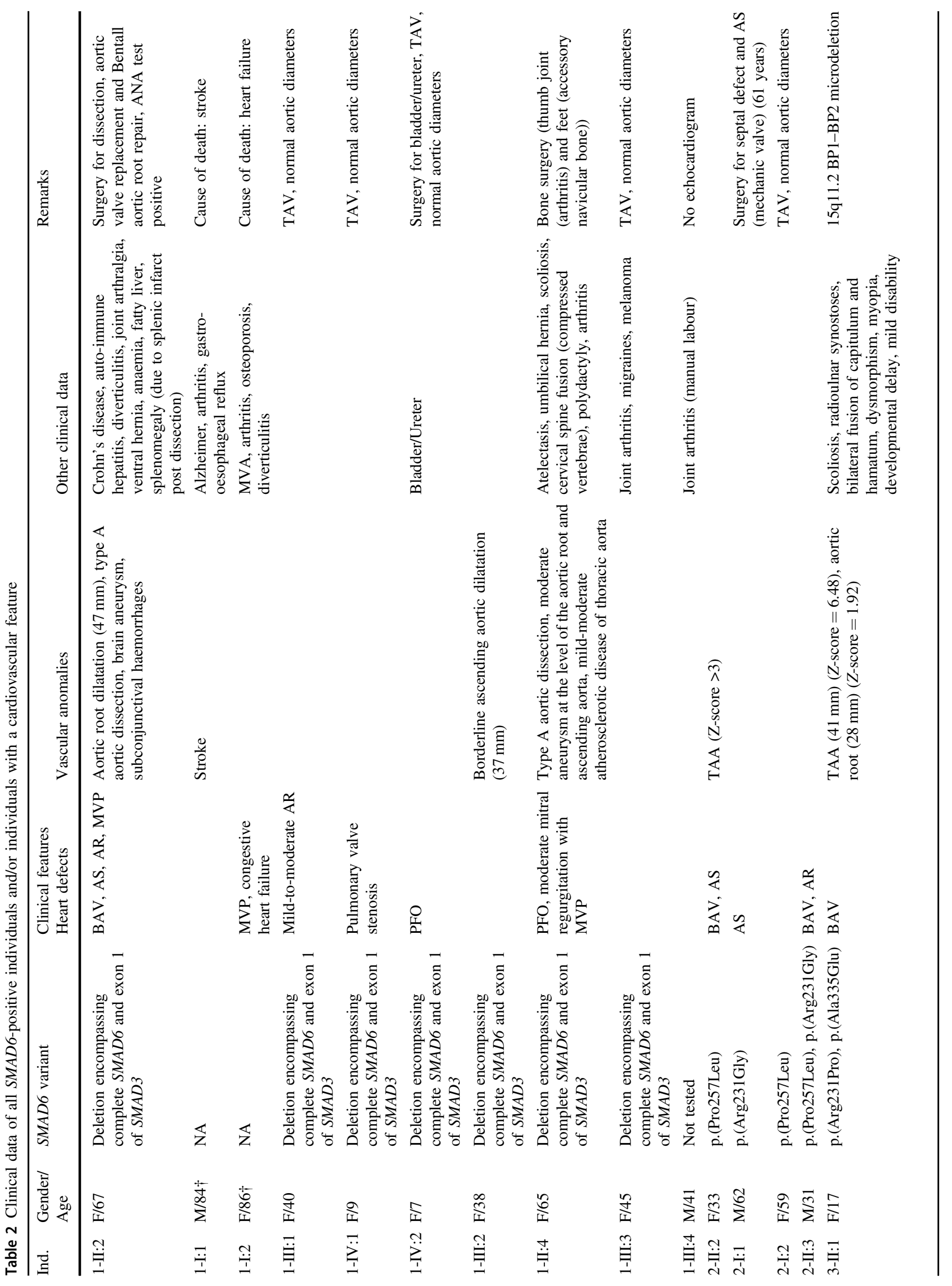




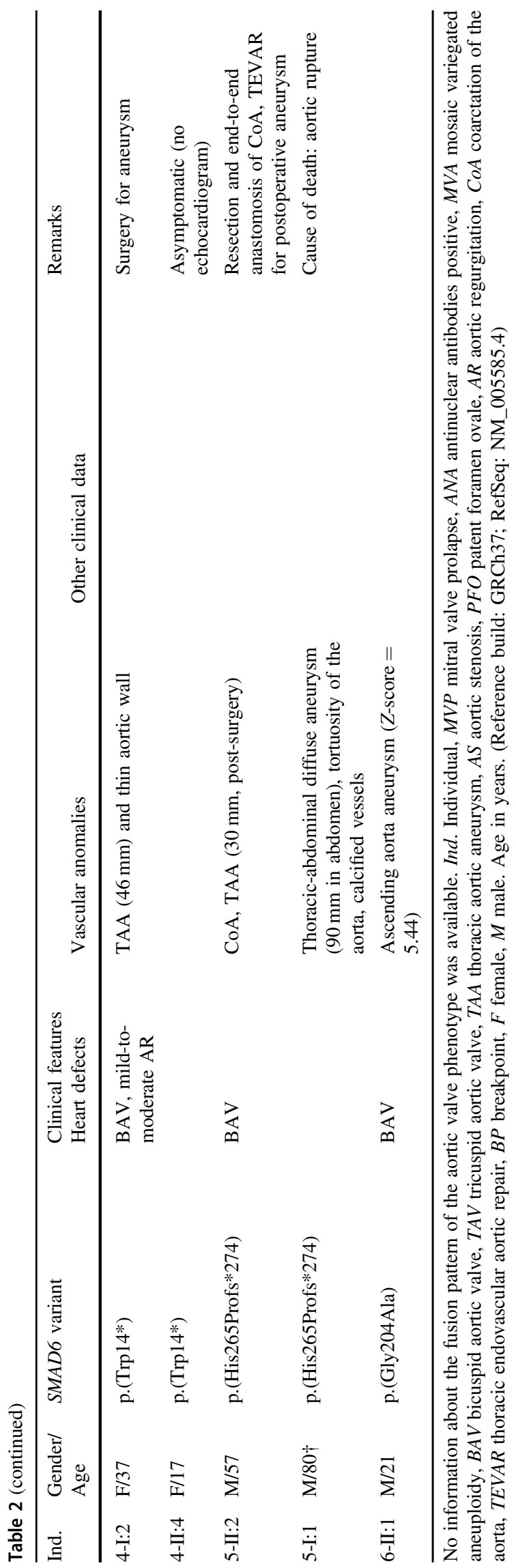

The proband of the second family, a 33-year-old female (2-II:2), carried the p.(Pro257Leu) SMAD6 variant. She was previously reported [9] and presented with BAV/TAA (Zscore $>3$ ) as well as mild aortic valve stenosis. A prototypical dilatation of the ascending aorta was observed as the diameter of the ascending aorta $(39 \mathrm{~mm})$ was bigger than the sinus of the aorta $(33 \mathrm{~mm})$. Surprisingly, another heterozygous missense variant of uncertain significance (c.691C > G, p.(Arg231Gly)) in the MH1-domain of SMAD6 was identified in the father (2-I:1; 62-year-old) of the proband (Fig. 1, Table 2). The p.(Arg231Gly) variant is absent from gnomAD and prediction programmes (PolyPhen-2, MutationTaster2 and SIFT) anticipate a deleterious effect with a CADD_phred score of 33 (Fig. 1). The father (2-I:1) underwent surgery (61 years) for a septal defect and received a mechanic valve prosthesis because of severe aortic stenosis. The unaffected 59-year-old mother of the proband (2-I:2) also carried the p.(Pro257Leu) variant. The proband's 31-year-old brother (2-II:3) presented with BAV and aortic regurgitation, and carries both SMAD6 variants. The unaffected 35-year-old sister (2-II:1) is variant-negative.

Interestingly, two SMAD6 variants were also identified in family 3 (Fig. 1, Table 2), i.e. p.(Arg231Pro) (c.692G > C, variant of uncertain significance) and p.(Ala335Glu) (c.1004C > A, likely pathogenic), located in the MH1- and MH2-domain, respectively. They are both absent from gnomAD, and a deleterious effect is predicted by different in silico programmes (Fig. 1) (PolyPhen-2, MutationTaster2 and SIFT) with a CADD_phred score of 30 for both variants. In addition to those two SMAD6 variants, the 17-yearold female proband (3-II:1) also carries a deletion between breakpoint site (BP) 1 and 2 on Chr15q11.2. She presented with a dysplastic asymmetric aortic valve with a hypoplastic left coronary leaflet and a progressive aortic dilatation of the ascending aorta $(41 \mathrm{~mm})(Z$-score $=6.48)$. The aortic root diameter at the level of the sinuses of Valsalva was normal $(28 \mathrm{~mm})(Z$-score $=1.92)$. Additionally, she displayed scoliosis, radioulnar synostoses, bilateral fusion of capitulum and hamatum, dysmorphism, myopia, developmental delay and mild disability. The latter neurological manifestations can most likely be explained by the Chr15q11.2 deletion.

The fifth identified SMAD6 variant was a nonsense pathogenic variant (c.42G > A, p.(Trp14*)), likely resulting in nonsense-mediated mRNA decay and, as such, haploinsufficiency. The variant is absent from gnomAD and has a CADD_phred score of 40 (Fig. 1). The 37-year-old female proband (4-I:2) displayed BAV with mild-to-moderate aortic regurgitation and an aneurysm of the aorta ascendens $(46 \mathrm{~mm})$ for which she underwent prophylactic aortic surgery in the context of the woman's desire for another pregnancy. Four of the proband's six children were checked for presence of the variant, of which one (4-II:4; 17 years) 
Table 3 Comparison of studies for evaluation of digenic model for craniosynostosis

\begin{tabular}{|c|c|c|c|c|}
\hline \multirow[t]{3}{*}{ Studies } & \multicolumn{4}{|c|}{ Carriers (probands) } \\
\hline & \multicolumn{2}{|c|}{$S M A D 6+/ B M P 2-$ risk allele } & \multicolumn{2}{|c|}{$S M A D 6+/ B M P 2+$ risk allele } \\
\hline & Craniosynostosis & No craniosynostosis & Craniosynostosis & No craniosynostosis \\
\hline Craniosynostosis cohort $^{\mathrm{a}}$ & $6(5)$ & $20(0)$ & $15(12)$ & $1(0)$ \\
\hline BAV/TAA cohort ${ }^{\mathrm{b}, \mathrm{c}}$ & $0(0)$ & $16(13)$ & $0(0)$ & $6(4)$ \\
\hline
\end{tabular}

${ }^{\mathrm{a} C}$ Combining all published papers [13, 14]

${ }^{\mathrm{b}}$ Combining this study with the published study of Gillis et al. [9]

${ }^{3}$ Of the 27 SMAD6-positive samples, 22 individuals were genotyped for the BMP2 risk allele because gDNA was not available for testing. The total number of the probands are shown between parenthesis

tested positive. She is asymptomatic. No gDNA of the two remaining children was available.

The next SMAD6 variant, i.e. c.794del (p.(His265Profs*274), pathogenic variant), is predicted to lead to a C-terminally elongated protein due to loss of the native stop codon. The variant is absent from gnomAD. The 57-year-old male proband (5-II:2) had BAV and CoA, which was treated with a resection and end-to-end anastomosis. Additionally, endovascular aortic repair was performed to treat a postoperative aneurysm of $30 \mathrm{~mm}$ located at the former $\mathrm{CoA}$ site. The proband's variant-positive father (5-I:1) deceased at age 80 from rupture of a thoracicabdominal diffuse aneurysm with a maximal abdominal diameter of $90 \mathrm{~mm}$. He was also known with aortic tortuosity and arterial wall calcification. No valve anomalies were reported.

Finally, we identified a missense variant located in the functional MH1-domain (c.611G > C, p.(Gly204Ala), variant of uncertain significance) that is absent from gnomAD, not unequivocally designated as pathogenic by prediction programs and has a CADD_phred score of 21.3. The male 21-year-old proband presented with BAV and an ascending aortic aneurysm $(Z$-score $=5.44)$, for which losartan treatment was started. Prior to this study, a class 3 splice region variant in FBN1 (c.5788+4C > A) was also identified in the proband [24]. This variant is reported five times in 245,778 alleles in the gnomAD database $(0.002 \%)$ and has a CADD_phred score of 9.4. With the exception of Human Splicing Finder, none of the other four prediction programs in Alamut, predicted the creation of a novel acceptor splicesite. Moreover, history and physical exam did only reveal surgery for a bilateral umbilical hernia but no other signs of connective tissue disease. Both parents are unavailable for clinical evaluation or genetic testing.

\section{Investigation of the phenotypic consequence of co-occurrence of a rare pathogenic SMAD6 variant and the BMP2 rs1884302 risk allele}

The SMAD6 variant-positive BAV/TAA patients reported previously by Gillis et al. and described here $(N=22)$ were investigated for the presence of the $B M P 2$ rs1884302 risk allele $\mathrm{C}(\mathrm{MAF}=34 \%$ [13]). The risk allele was identified in 6 out of the 22 SMAD6-positive BAV/TAA patients $(N=6 /$ $22,27.2 \%)$. None of our SMAD6+/BMP2+ BAV/TAA patients presented with craniosynostosis (retrospective analysis) (Table 3). Although there are only limited data on the fusion pattern of the aortic valve and the location of the aneurysm, no trend for a difference between $B M P 2+$ and $B M P 2$ - SMAD6-variant-positive patients in our cohort was observed.

\section{Discussion}

Loss-of-function variants in SMAD6 have previously been shown to cause both CHD [9-11] and craniosynostosis $[13,14]$. SMAD6 encodes a negative regulator of the BMP signalling pathway that plays an important role in aortic valve development and skull formation [26, 27]. In a nonsyndromic TAA cohort consisting of 473 patients, we found seven novel likely pathogenic SMAD6 variants in six families, including one family that was previously reported by Gillis et al. [9]. Although only $14 \%$ of our patients in our cohort has a BAV in combination with TAA, all probands carrying likely pathogenic SMAD6 variants presented with BAV/TAA $(N=7 / 65,10.8 \%)$. Thus, our study confirms the prominent contribution of genetic variability in SMAD6 to the aetiology of BAV/TAA. Rare SMAD6 variants were not identified in pure TAV/TAA families, suggesting a limited role for SMAD6 genetic variability in TAV/TAA disease. Previously, SMAD6 variants were reported to explain $2.5 \%$ of BAV/TAA patients [9]. The higher frequency (10.8\%) of SMAD6 variants in this BAV/TAA cohort might be attributed to the more severe cardiovascular phenotype, demonstrated by a younger inclusion age $(47.6 \pm 17.4$ years versus $63.5 \pm 14.4$ years) and a higher proportion of positive family histories (30\% versus $9 \%$ ). The positive predictive value of a family history for cardiovascular disease is further supported by the observation that $50 \%$ of the SMAD6-positive patients in this cohort presented with a positive family history $(N=3 / 6)$. 
Similarly to the published rare deleterious SMAD6 variants, we also identified nonsense, frameshift and missense SMAD6 variants locating in the MH1/MH2-domain of the protein. Furthermore, we identified the first SMAD6 gene deletion. SMAD6 deletions have not yet been reported in the DECIPHER database nor in patients with craniosynostosis $[13,14]$. The deleted region also included exon 1 of $S M A D 3$ and, as such, results in haploinsufficiency for SMAD3. SMAD3 loss-of-function mutations cause LoeysDietz syndrome type 3, a TAA syndrome characterized by MVP, aortic regurgitation, hernias and arterial aneurysms [28]. An increased prevalence of BAV is also observed in patients with Loeys-Dietz syndrome type 3 [29]. We cannot exclude the possibility that this SMAD3 deletion also contributes to the observed cardiovascular phenotype.

Besides variable BAV and TAA expressivity, segregation analysis also revealed incomplete penetrance as well as the occasional occurrence of extra-cardiovascular features. In our current study, 17 SMAD6-variant carriers were identified in six SMAD6-positive families, from which only the probands (average age of 38.7 years) presented with BAV/TAA $(N=6 / 17,35.3 \%)$. Non-penetrance for cardiovascular features was observed in 3/17 SMAD6-variant carriers (average age of 40 years; 1-III:3, 2-I:2 and 4-II:4) $(N=3 / 17,17.6 \%)$. In total, cardiovascular manifestations were observed in 14 SMAD6-variant carriers $(N=14 / 17$, $82.4 \%$ ). In the probands, two presented with BAV/TAA only whereas four BAV/TAA patients also presented with aortic valve stenosis (1-II:2, 2-II:2), aortic regurgitation (1-II:2, 4-I:2), MVP (1-II:2), brain aneurysm (1-II:2), subconjunctival haemorrhages (1-II:2) and CoA (5-II:2). The latter BAV/TAA patient (5-II:2) was treated for CoA with surgery. Intriguingly, $\mathrm{CoA}$ was described in two SMAD6-related BAV(/TAA) families before [9, 11]. Even though the total number of SMAD6 variant-positive patients with CHD is still relatively low $(N=30)$, a trend towards a higher prevalence of, and hence increased risk for, CoA $(10 \%)$ is observed as compared to (BAV/TAA) cases in general (7\%) [5]. Of the remaining eight SMAD6-positive variant carriers, six displayed a heart defect including BAV (2-II:3), aortic regurgitation (1-III:1, 2-II:3), aortic valve stenosis (2-I:1), which was treated with surgery, patent foramen ovale (1-IV:2, 1-II:4), moderate mitral regurgitation with MVP (1-II:4), and pulmonary valve stenosis (1IV:1). Additionally, one of these patients (1-II:4) presented with vascular anomalies like type A aortic dissection and moderate aneurysm at the level of the aortic root and ascending aorta, which is potentially aggravated by mildmoderate atherosclerosis. Finally, two out of the 14 SMAD6variant carriers only presented vascular anomalies. One patient (5-1:1) had a diffuse thoracic-abdominal aneurysm, tortuosity of the aorta, and calcified arterial vessels, while the other patient (1-III:2) presented with a mild aortic dilatation.
Non-cardiovascular disease was found in five out of the 17 SMAD6-positive patients $(N=5 / 17,29.4 \%)$. However, four out of these five patients $(N=4 / 5,80 \%)$ belonged to the same family and harboured, in addition to the SMAD6 deletion, a deletion of SMAD3 exon 1. The clinical features of these patients presented in various systems such as the musculoskeletal system (1-II:2, 1-II:4, 1-III:3), the central nerve system (1-III:3), the immune system (1-II:2, 1-II:4) and the genitourinary system (1-IV:2). The fifth patient (3-II:1) presented extra features like mild disability and developmental delay, most likely accounted for by the Chr15q11.2 microdeletion, as discussed above. Overall, our data suggest that the phenotypical expression of SMAD6 variants seems confined to the cardiovascular system. Taken together, these observations makes genetic counselling of SMAD6-positive patients quite difficult.

Since both cardiovascular features and craniosynostosis are observed as the consequence of an identical type of variant (i.e. loss-of-function), it is tempting to hypothesize that other genetic factors could be involved in modifying the phenotypic outcome of SMAD6 deficiency. In five out of our six BAV/TAA families, a second genetic class 3, 4 or 5 variant was identified, which could potentially increase the penetrance for CHD. Two SMAD6-positive families (family 2 and 3) harboured a second rare SMAD6 missense variant located in a functional domain. Interestingly, in both families one of the variants affected the amino acid at position 231. Unfortunately, segregation analysis was only performed in family 2 , but the patient harbouring both SMAD6 variants did not present with a strikingly more severe cardiovascular phenotype. Moreover, family members presenting either of the two SMAD6 variants also had a cardiovascular phenotype, suggesting that each of them is at least contributing to the phenotype. Also, two SMAD6positive families carried a second hit in another TGF $\beta$ related gene, i.e. a deletion of exon 1 of SMAD3 or a potential splice-site variant in FBN1. Another family had a Chr15q11.2 microdeletion in addition to the SMAD6 missense variant. BAV/TAA has not been reported with the 15q11.2 BP1-BP2 microdeletion syndrome but few patients [30] have been described with cardiovascular defects, including ventricular septal defect, patent ductus arteriosus and patent foramen ovale [31, 32].

Current insights into the molecular disease mechanisms of craniosynostosis were solely acquired by studies with patients displaying this skull phenotype, in which incomplete penetrance for rare deleterious SMAD6 variants was observed. However, near-complete penetrance was reached upon co-occurrence with a variant near BMP2 (rs1884302) $[13,14]$. Hence, absence of this common variant was expected in our SMAD6-variant-positive study population. We identified six $S M A D 6+/ B M P 2+$ non-syndromic TAA patients without craniosynostosis. This is not in line with 
the findings by Timberlake et al. $[13,14]$. They showed the presence of the $B M P 2$ SNP risk allele in 16 out of their 41 SMAD6-positive individuals $(N=16 / 41,39 \%)$ and only 1 out of the 16 individuals with the pathogenic SMAD6+/ $B M P 2+$ genotype did not present craniosynostosis $(6 \%)$ (Table 3) [13, 14]. As such, we were unable to support the proposed digenic model described by Timberlake et al. [13]. Our findings suggest involvement of additional factors that contribute to the phenotypic outcome of patients either causing CHD or craniosynostosis. Supportive evidence for these observations is inferred by mouse studies. Mice depleted for the murine orthologue of SMAD6, i.e. $M a d h 6^{-1-}$ mice, presented with a sole cardiovascular [33] (129/SvEv $\times$ BALB/cBy; $129 / \mathrm{SvEv} \times \mathrm{C} 57 \mathrm{~B} 1 / 6 ; \quad 129 / \mathrm{SvEv}$ inbred) or craniofacial, axial and appendicular skeletal phenotype [34] $(\mathrm{C} 57 \mathrm{~B} 1 / 6 \times \mathrm{BALB} / \mathrm{c})$ depending on the genetic background of the mouse model. This supports the need for looking into the genetic backgrounds of these patients. Ultimately, more research focussing on gaining insights into the diseases' characteristics will allow better risk stratification strategies as well as patient management. Limitations of our study include the need for further functional validation of the identified SMAD6 variants and an additional unbiased study to address modifying risk factors that influence the cardiovascular phenotypes of SMAD6variant-positive individuals.

To conclude, our results confirm the role of deleterious SMAD6 variants in the genetic aetiology of the BAV/TAA pathology, and reveal little contribution to TAV/TAA development. Besides variable clinical expressivity, reduced penetrance and the occasional occurrence of extra cardiovascular features, an increased risk for CoA seems to be emerging. The proposed digenic model of near-complete penetrance for craniosynostosis was not supported by findings in our BAV/TAA cohort, suggesting that other genetic or stochastic factors are at play. Finally, our data provide novel insights into the complex genetic architecture of (BAV/)TAA disease with important implications for molecular diagnostics. Screening of SMAD6 in young BAV/TAA patients, especially with a positive history for $\mathrm{CHD}$, is recommended.

Acknowledgements We would like to thank the patients and their families for their willingness to participate in the study.

Funding This research was supported by funding from the University of Antwerp (Lanceringsproject), the Fund for Scientific Research, Flanders (FWO, Belgium, G.0356.17), The Dutch Heart Foundation (2013T093) and the Foundation Leducq (MIBAVA-Leducq 12CVD03). LB is senior clinical investigator of the Fund for Scientific Research, Flanders and holds a starting grant from the European Research Council (ERC-StG-2012-30972-BRAVE). VG and VA are postdoc researchers and supported by the Fund for Scientific Research Flanders. MJ is supported by the Fund for Scientific Research
Flanders. LI is supported by a PhD grant from the Agency for Innovation by Science and Technology (IWT).

\section{Compliance with ethical standards}

Conflict of interest The authors declare that they have no conflict of interest.

Publisher's note: Springer Nature remains neutral with regard to jurisdictional claims in published maps and institutional affiliations.

\section{References}

1. Criado FJ. Aortic dissection: a 250-year perspective. Tex Heart Inst J. 2011;38:694-700.

2. Andelfinger G, Loeys B, Dietz H. A decade of discovery in the genetic understanding of thoracic aortic disease. Can J Cardiol. 2016;32:13-25.

3. Braverman AC, Guven H, Beardslee MA, Makan M, Kates AM, Moon MR. The bicuspid aortic valve. Curr Probl Cardiol. 2005;30:470-522.

4. Edwards WD, Leaf DS, Edwards JE. Dissecting aortic aneurysm associated with congenital bicuspid aortic valve. Circulation. 1978;57:1022-5.

5. Michelena HI, Khanna AD, Mahoney D, Margaryan E, Topilsky $\mathrm{Y}$, Suri RM et al. Incidence of aortic complications in patients with bicuspid aortic valves. JAMA. 2011;306:1104-12.

6. Bowdin SC, Laberge AM, Verstraeten A, Loeys BL. Genetic testing in thoracic aortic disease-when, why, and how?. Can J Cardiol. 2016;32:131-4.

7. Brownstein AJ, Ziganshin BA, Kuivaniemi H, Body SC, Bale AE, Elefteriades JA. Genes associated with thoracic aortic aneurysm and dissection: an update and clinical implications. Aorta (Stamford). 2017;5:11-20.

8. Giusti B, Sticchi E, De Cario R, Magi A, Nistri S, Pepe G. Genetic bases of bicuspid aortic valve: the contribution of traditional and high-throughput sequencing approaches on research and diagnosis. Front Physiol. 2017;8:612

9. Gillis E, Kumar AA, Luyckx I, Preuss C, Cannaerts E, van de Beek $\mathrm{G}$ et al. Candidate gene resequencing in a large bicuspid aortic valve-associated thoracic aortic aneurysm cohort: SMAD6 as an important contributor. Front Physiol. 2017;8:400.

10. Jin SC, Homsy J, Zaidi S, Lu Q, Morton S, DePalma SR et al. Contribution of rare inherited and de novo variants in 2,871 congenital heart disease probands. Nat Genet. 2017;49:1593-601.

11. Tan HL, Glen E, Töpf A, Hall D, O'Sullivan JJ, Sneddon L et al. Nonsynonymous variants in the SMAD6 gene predispose to congenital cardiovascular malformation. Hum Mutat. 2012;33:720-7.

12. Hata A, Lagna G, Massague J, Hemmati-Brivanlou A. Smad6 inhibits BMP/Smad1 signaling by specifically competing with the Smad4 tumor suppressor. Genes Dev. 1998;12:186-97.

13. Timberlake AT, Choi J, Zaidi S, Lu Q, Nelson-Williams C, Brooks ED et al. Two locus inheritance of non-syndromic midline craniosynostosis via rare SMAD6 and common BMP2 alleles. eLife 2016;5.

14. Timberlake AT, Furey CG, Choi J, Nelson-Williams C. De novo mutations in inhibitors of Wnt, BMP, and Ras/ERK signaling pathways in non-syndromic midline craniosynostosis. Proc Natl Acad Sci USA. 2017;114:E7341-7.

15. Devereux RB, de Simone G, Arnett DK, Best LG, Boerwinkle E, Howard BV et al. Normal limits in relation to age, body size and gender of two-dimensional echocardiographic aortic root 
dimensions in persons $>/=15$ years of age. Am $\mathrm{J}$ Cardiol. 2012;110:1189-94.

16. Warren AE, Boyd ML, O'Connell C, Dodds L. Dilatation of the ascending aorta in paediatric patients with bicuspid aortic valve: frequency, rate of progression and risk factors. Heart. 2006;92:1496-1500.

17. Proost D, Vandeweyer G, Meester JA, Salemink S, Kempers M, Ingram $\mathrm{C}$ et al. Performant mutation identification using targeted next-generation sequencing of 14 thoracic aortic aneurysm genes. Hum Mutat. 2015;36:808-14.

18. O'Roak BJ, Vives L, Fu W, Egertson JD, Stanaway IB, Phelps IG et al. Multiplex targeted sequencing identifies recurrently mutated genes in autism spectrum disorders. Science. 2012;338:1619-22.

19. Lek M, Karczewski KJ, Minikel EV, Samocha KE, Banks E, Fennell $\mathrm{T}$ et al. Analysis of protein-coding genetic variation in 60,706 humans. Nature. 2016;536:285-91.

20. Kircher M, Witten DM, Jain P, O'Roak BJ, Cooper GM, Shendure J. A general framework for estimating the relative pathogenicity of human genetic variants. Nat Genet. 2014; 46:310-5.

21. Schwarz JM, Cooper DN, Schuelke M, Seelow D. MutationTaster2: mutation prediction for the deep-sequencing age. Nat Methods. 2014;11:361-2.

22. Ng PC, Henikoff S. Predicting deleterious amino acid substitutions. Genome Res. 2001;11:863-74.

23. Adzhubei IA, Schmidt S, Peshkin L, Ramensky VE, Gerasimova A, Bork P et al. A method and server for predicting damaging missense mutations. Nat Methods. 2010;7:248-9.

24. Richards S, Aziz N, Bale S, Bick D, Das S, Gastier-Foster J et al. Standards and guidelines for the interpretation of sequence variants: a joint consensus recommendation of the American College of Medical Genetics and Genomics and the Association for Molecular Pathology. Genet Med. 2015;17:405-24.

25. LeMaire SA, Russell L. Epidemiology of thoracic aortic dissection. Nat Rev Cardiol. 2011;8:103-13.
26. Imamura T, Takase M, Nishihara A, Oeda E, Hanai J, Kawabata $\mathrm{M}$ et al. Smad6 inhibits signalling by the TGF-beta superfamily. Nature. 1997;389:622-6.

27. Wang Y, Ho CC, Bang E, Rejon CA, Libasci V, Pertchenko P et al. Bone morphogenetic protein 2 stimulates noncanonical SMAD2/3 signaling via the BMP type $1 \mathrm{~A}$ receptor in gonadotrope-like cells: implications for FSH synthesis. Endocrinology. 2014;155:1970-81.

28. van de Laar IM, Oldenburg RA, Pals G, Roos-Hesselink JW, de Graaf BM, Verhagen JM et al. Mutations in SMAD3 cause a syndromic form of aortic aneurysms and dissections with earlyonset osteoarthritis. Nat Genet. 2011;43:121-6.

29. van de Laar IM, van der Linde D, Oei EH, Bos PK, Bessems JH, Bierma-Zeinstra SM et al. Phenotypic spectrum of the SMAD3related aneurysms-osteoarthritis syndrome. J Med Genet. 2012;49:47-57.

30. Cox DM, Butler MG. The 15q11.2 BP1-BP2 microdeletion syndrome: a review. Int J Mol Sci. 2015;16:4068-82.

31. Doornbos M, Sikkema-Raddatz B, Ruijvenkamp CA, Dijkhuizen T, Bijlsma EK, Gijsbers AC et al. Nine patients with a microdeletion 15q11.2 between breakpoints 1 and 2 of the Prader-Willi critical region, possibly associated with behavioural disturbances. Eur J Med Genet. 2009;52:108-15.

32. Wong D, Johnson SM, Young D, Iwamoto L, Sood S, Slavin TP. Expanding the BP1-BP2 15q11.2 microdeletion phenotype: tracheoesophageal fistula and congenital cataracts. Case Rep Genet. 2013;2013:801094

33. Galvin KM, Donovan MJ, Lynch CA, Meyer RI, Paul RJ, Lorenz $\mathrm{JN}$ et al. A role for smad6 in development and homeostasis of the cardiovascular system. Nat Genet. 2000;24:171-4.

34. Estrada KD, Retting KN, Chin AM, Lyons KM. Smad6 is essential to limit BMP signaling during cartilage development. J Bone Miner Res. 2011;26:2498-510.

\section{Affiliations}

Ilse Luyckx ${ }^{1} \cdot$ Gretchen MacCarrick $^{2} \cdot$ Marlies Kempers $^{3} \cdot$ Josephina Meester $^{1} \cdot$ Céline Geryl $^{1} \cdot$ Olivier Rombouts $^{1}$. Nils Peeters ${ }^{1}$ - Charlotte Claes $^{1}$ - Nele Boeckx ${ }^{1}$ - Natzi Sakalihasan ${ }^{4}$ - Adeline Jacquinet ${ }^{5}$ - Alexander Hoischen ${ }^{3,6,7}$. Geert Vandeweyer $^{1}$. Sarah Van Lent ${ }^{1} \cdot$ Johan Saenen $^{8}$. Emeline Van Craenenbroeck ${ }^{8}$. Janneke Timmermans ${ }^{9}$. Anthonie Duijnhouwer $\mathbb{D}^{9} \cdot$ Harry Dietz ${ }^{2,10,11,12} \cdot$ Lut Van Laer $^{1} \cdot$ Bart Loeys $^{1,3} \cdot$ Aline Verstraeten $^{1}$

1 Center of Medical Genetics, Faculty of Medicine and Health Sciences, University of Antwerp and Antwerp University Hospital, Antwerp, Belgium

2 McKusick-Nathans Institute of Genetic Medicine, Johns Hopkins University School of Medicine, Baltimore, MD, USA

3 Department of Human Genetics, Radboud University Nijmegen Medical Center, Nijmegen, The Netherlands

4 Surgical Research Center, GIGA-R, Belgium; Department of Cardiovascular and Thoracic Surgery, University Hospital of Liège, Liège, Belgium

5 Center for Human Genetics, Centre Hospitalier Universitaire and University of Liège, Liège, Belgium

6 Radboud Institute of Molecular Life Sciences, Radboud University Nijmegen Medical Center, Nijmegen, The Netherlands
7 Department of Internal Medicine and Radboud Center for Infectious Diseases (RCI), University Nijmegen Medical Center, Nijmegen, The Netherlands

8 Department of Cardiology, University of Antwerp, Antwerp University Hospital, Antwerp, Belgium

9 Department of Cardiology, Radboud University Hospital, Nijmegen, The Netherlands

10 Howard Hughes Medical Institute, Baltimore, MD, USA

11 Department of Pediatrics, Division of Pediatric Cardiology, Johns Hopkins University School of Medicine, Baltimore, MD, USA

12 Department of Medicine, Johns Hopkins University School of Medicine, Baltimore, MD, USA 